\title{
A case for restricted-domain relational learning
}

\author{
ANTHONY A. WRIGHT \\ University of Texas Health Science Center at Houston, Houston, Texas \\ AND \\ JEFFREY S. KATZ \\ Auburn University, Auburn, Alabama
}

\begin{abstract}
Monkeys and pigeons learned a same/different task with pairs that were selected from a training set of eight picture stimuli. They showed no novel-stimulus transfer and hence no abstract-concept learning. They were also tested with novel pairs of the eight training pictures (i.e., combinations that had not been used in training) and with inverted pictures of the training pairs. If the subjects had learned the task item-specifically (e.g., if-then or configural learning), they should have failed these tests, but they performed well with novel combinations of training pictures and inverted pictures, suggesting that they learned the task relationally (i.e., on the basis of the relationship between the two pictures that were presented in each trial). This somewhat paradoxical conclusion of relational learning in the absence of abstract-concept learning is contrary to most theories of abstract-concept learning. The implications of this conclusion are discussed in the context of restricted-domain relational learning.
\end{abstract}

Children initially process the visual domain in terms of specific item features and later transition to relationships between or among different visual stimuli (see, e.g., Doumas, Hummel, \& Sandhofer, 2008; Gentner, 2003; Gentner \& Rattermann, 1991). Relational learning begins as global similarity (i.e., identity), but it can later extend to shared attributes (see, e.g., Siegler \& Alibali, 2004; Smith, 1984 , 1989). The transfer of relational learning to novel stimuli has been the hallmark of abstract-concept learning, and it is based on an overarching relational rule that is applied to pairs of novel stimuli.

Failures of novel-item relational transfer have been taken not only as evidence against abstract-concept learning, but also as evidence for item-specific learning. This assumption of item-specific learning when concept learning fails has a long history in determining which species have the cognitive capability to learn abstract concepts (see, e.g., Carter \& Werner, 1978; Premack, 1978, 1983a, 1983b; Thomas, 1980). Carter and Werner (1978) and Premack (1978) elaborated on Skinner's (1950) suggestion that pigeons might learn specific stimulus-response chains, or if-then associations, for each sample and correct comparison choice, and on Lashley's (1938) suggestion that animals might learn configural patterns of stimulus combinations (i.e., gestalt configurations). Why these hypotheses remained untested for so many decades is a bit of a mystery. Equally puzzling is why these untested hypotheses were accepted as fact (fait accompli) for so many decades.

Our first indication that something might be amiss in assuming item-specific learning when novel-stimulus transfer fails came from studies in which pigeons, rhesus monkeys, and capuchin monkeys learned a same/different (S/D) task with 8 picture items but showed no novelstimulus transfer (Katz \& Wright, 2006; Katz, Wright, \& Bachevalier, 2002; Wright \& Katz, 2006; Wright, Rivera, Katz, \& Bachevalier, 2003). If these subjects had learned the task item-specifically, then in a series of training-set expansions $(16,32,64,128$, etc.), they should at some point have switched from item-specific learning to relational learning and shown a sudden increase in novelstimulus transfer. There was no sudden increase or discontinuity in the novel-stimulus transfer function, however; novel-stimulus transfer increased at a steady (linear) rate with training-set doublings.

On the one hand, these abstract-concept learning results were encouraging: Abstract-concept learning was found across pigeons and two different monkey species. On the other hand, these results were puzzling. If these species did not learn the S/D task item-specifically when they failed the test for abstract-concept learning, what did they learn?

The purpose of the present research was to train other groups of pigeons and monkeys in a similar eight-item S/D task and then test them for item-specific learning when they failed to show abstract-concept learning. One test was with stimulus pairs that were set aside for testing following acquisition. The other test was with inverted stimuli of the training pairs. The rationale was that these tests would likely disrupt item-specific learning but would preserve relational learning, because those picture pairs would still be the same or different.

A. A.Wright, anthony.a.wright@uth.tmc.edu 


\section{METHOD}

\section{Subjects}

The subjects were four, 2- to 6-year-old naive White Carneaux pigeons (Columba livia) and four, 4- to 8-year-old, naive male rhesus monkeys (Macaca mulatta). Testing was conducted 5 days per week. Monkeys had access to Purina Monkey Chow and water in home cages for $8 \mathrm{~h}$ after testing and to fruits, vegetables, monkey biscuits, and water on weekends. Pigeons were maintained at $85 \%$ of their free-feeding weights and had access to grit and water in home cages. A 14:10-h light:dark cycle was maintained in colony rooms, and animals were tested during the light cycle.

\section{Apparatus}

Monkeys were tested unrestrained in custom aluminum test chambers. The reinforcer was either a $300-\mathrm{mg}$ banana pellet or $5 \mathrm{cc}$ of Tang orange drink. Pigeons were tested in custom wooden test chambers. For both species, 40.3-cm Eizo color monitors, Carroll touch screens, Metrabyte relay interfaces, Pentium PCs, and custom Visual Basic software were used to control the experiments. For monkeys, digitized pictures $(13.8 \times 9.7 \mathrm{~cm})$ were presented at $800 \times 600$ pixel resolution, on a black background with a $3.4-\mathrm{cm}$ gap, with a white different response area $(6.25 \times 5.6 \mathrm{~cm})$ that was aligned with the bottom picture and a $3.7-\mathrm{cm}$ gap, and with a Plexiglas template to eliminate paw swiping. Display dimensions were reduced by $60 \%$ for pigeons to equate visual angles.

\section{Stimuli}

Figure 1 shows the 40 training pairs ( 8 same, 32 different) and 24 testing pairs (untrained set). The eight training pictures appeared equally often in top and bottom positions on both same and different trials, so that individual stimuli could not cue the correct choice response. For this reason, none of the same pairs of stimuli could be reserved for testing following acquisition, because those pictures would have been associated with different trials only and could have cued "different" responses. The eight training pictures also appeared equally often in top and bottom positions on testing pairs.

\section{Procedure}

Training for monkeys was similar to that for pigeons. Responses were shaped by successive approximations or were autoshaped (one to seven sessions) to white rectangles (successively presented) in the different response area and in the lower picture position. S/D training began with presentation of the upper picture. Following a single touch or peck, the lower picture and the white rectangle were presented simultaneously along with the upper picture. If the two pictures were the same, a touch or peck to the lower picture was correct and was rewarded. If the two pictures were different, a touch or peck to the white rectangle was correct and was rewarded. After the choice response, the display was extinguished. A correct choice resulted in a 0.5 -sec tone and reinforcement: banana pellet or Tang for monkeys, and mixed grain for pigeons. The amount of drink or grain varied on an individual subject basis. Incorrect choices were not reinforced. Choices were followed by a 15 -sec intertrial interval (ITI). Starting on the fifth training session, incorrect choices were followed by correction procedure: a darkened 15 -sec timeout, the ITI, and repetition of the incorrect trial. The results that are presented here were based on first-trial performance only. Responses to the upper picture were increased to 10 for monkeys and 20 for pigeons over Sessions 1-15. Sessions were 96 pseudorandom trials (48 same, 48 different), and stimulus pairs were counterbalanced to the degree possible. When performance reached the criterion of $80 \%$ correct or better for three consecutive sessions, the correction procedure was removed and the criterion was reestablished.

Untrained-set testing. Following acquisition, transfer to the 24 testing pairs was tested for 20 consecutive sessions. Each test session contained 96 trials. There were 84 baseline/training trials (48 same, 36 different) and 12 transfer trials. Test pairs were counterbalanced over 2 test sessions and were pseudorandomly placed in test sessions following Trial 7. Correct responses on transfer trials were reinforced in order to avoid having subjects learn that the test pairs signaled no reinforcement or extinction.

Novel-stimulus testing. Following untrained-set testing, novelstimulus testing was conducted for six consecutive sessions. Each testing session contained 100 trials ( 90 baseline trials, 5 same transfer trials, and 5 different transfer trials; see Wright \& Katz, 2006, Figure 4, for the novel test pairs that were used). Correct performance was reinforced in the same manner as on baseline trials.

Stimulus-inversion testing. Transfer to training pairs with the stimulus pictures turned upside down (but maintaining their original top and bottom positions) was tested for five consecutive sessions. Each test session contained 96 trials ( 86 baseline trials, 5 same transfer trials, and 5 different transfer trials) with random selection without replacement. Correct responses on transfer trials were reinforced.

\section{RESULTS AND DISCUSSION}

\section{Acquisition}

The monkeys and pigeons acquired the task in 61 and 22 (mean) sessions, respectively. The slowest monkey to acquire responded very rapidly and changed its choice response so rapidly that the response template had to be adjusted (moved out) to slow responding and make responses more deliberate. The adjustment immediately enhanced this monkey's acquisition (and that of others as well), but it may have contributed to variability in the monkeys' acquisition. In any case, the group acquisition rates for monkeys and pigeons were not significantly different $[F(1,6)=4.1, p=.09]$. All subjects showed some differences in same versus different performances, which often reversed early in acquisition but dissipated as the task was acquired. At the point at which the subjects met criterion (last training day), there was no difference within or across species between same (pigeon, 90.8\%; rhesus, $87.5 \%$ ) and different (pigeon, $79.7 \%$; rhesus, $87.5 \%$ ) performance, as confirmed by a two-way repeated measures ANOVA of trial type (same, different $) \times$ species, which yielded no main effects of trial type $[F(1,6)=2.4, p>.17]$ or species $[F(1,6)<1]$ and no interaction $[F(1,6)=2.4, p>.17]$.

Learning rates might have been affected by how the task was learned; for example, learning individual item pairs (item-specific learning) might be advantageous initially, but learning relationships might win out in the end. Learning rate in the present task was thus compared with the learning rates of 3 monkeys and 4 pigeons that were trained in the same task but with all 56 different pairs (Katz \& Wright, 2006; Katz et al., 2002). Learning rates (trials to criterion) in these two tasks were found to be equivalent, as shown by a two-way ANOVA of species $\times$ group (untrained set vs. no untrained set) (all $F_{\mathrm{s}}<3.6$, all $p \mathrm{~s}>.31)$.

The similarity in learning rates in these two tasks is important, because it shows that the removal of 24 counterbalanced pairs for later testing in the present experiment did not alter the number of trials or sessions to learn the task. Indeed, this result in itself argues against itemspecific learning. If the subjects had learned each stimulus pair item-specifically, then trials to criterion in the present task should have been proportionally less $(37 \%)$, because fewer pairs had to be learned. But there was no savings 


\section{Training Set}

8 Same Pairs
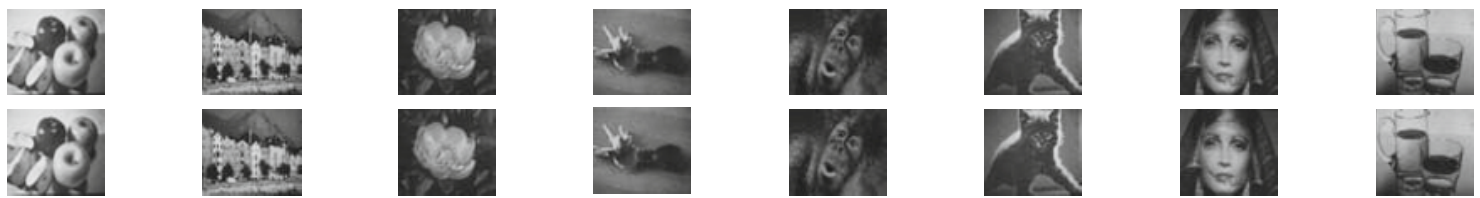

32 Different Pairs
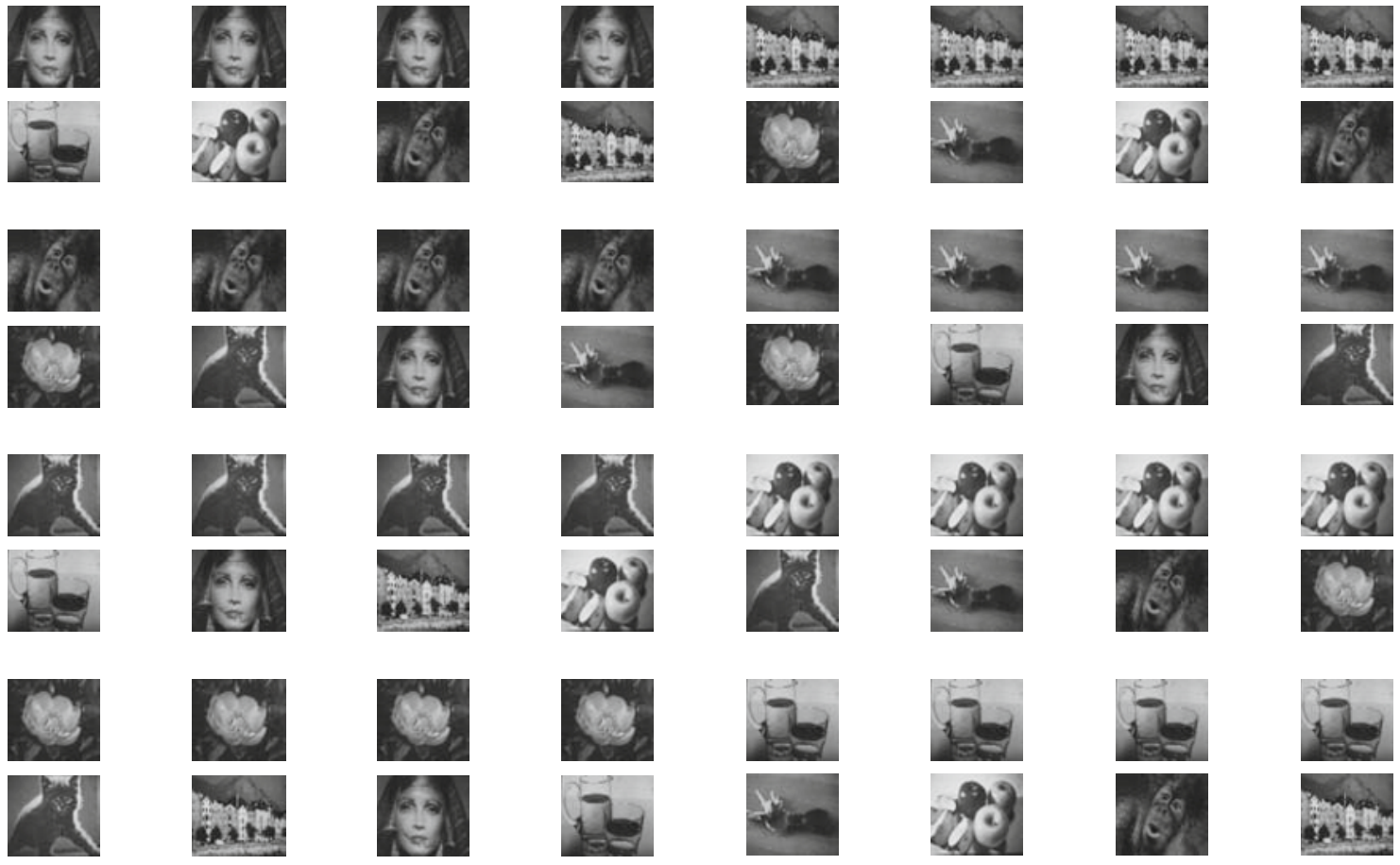

\section{Untrained Set}
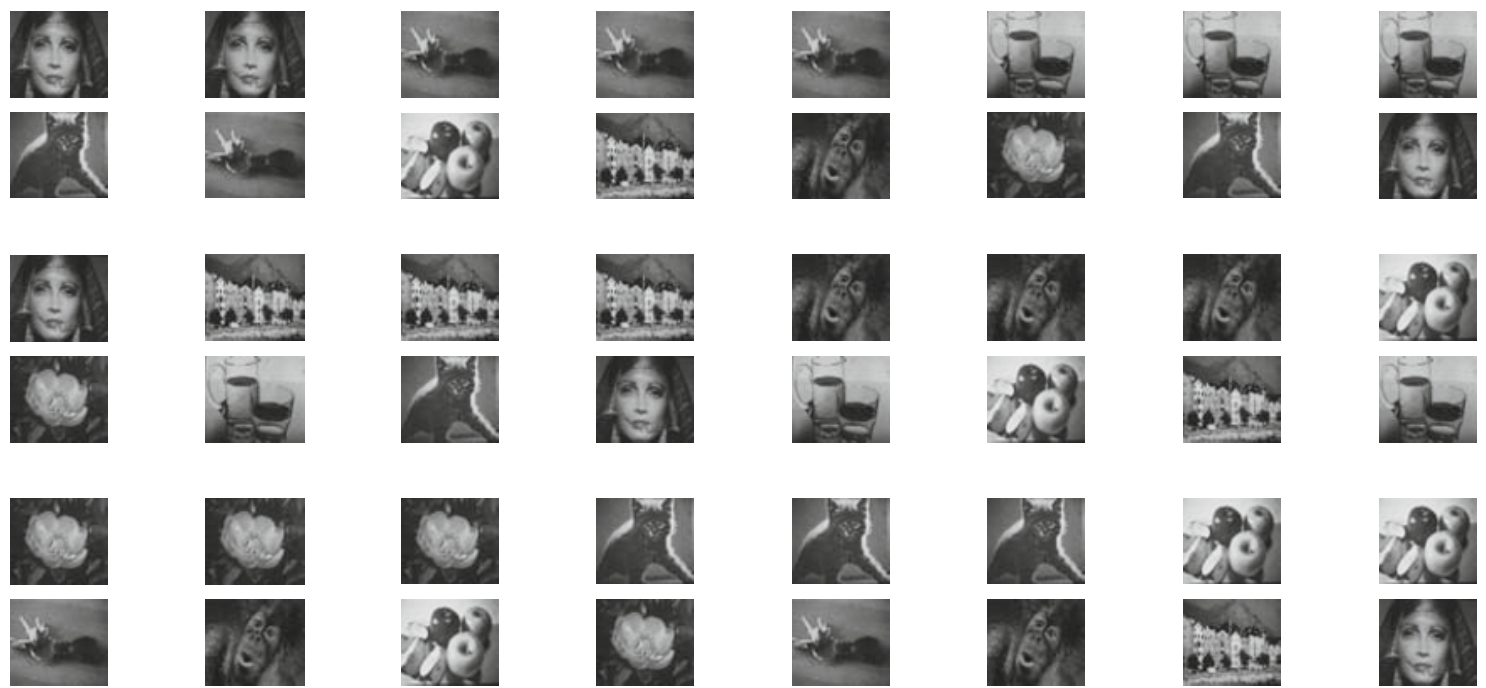

Figure 1. Top: Training set of 8 same pairs and 32 different pairs, with each picture appearing four times in the upper position and four times in the lower position. Bottom: Untrained set of 24 counterbalanced pairs, with each picture appearing three times in each position. (See Wright \& Katz, 2006, Figure 2, for these pairs printed in color.) 
with fewer pairs to be learned in the present experiment, suggesting that these tasks were being learned relationally, not item-specifically.

\section{Untrained-Set Transfer}

Following acquisition, the pigeons and monkeys were tested with the untrained stimulus pairs, and these results are shown in Figure 2. If the subjects had learned each training pair as a unique pair, performance with the test pairs of the untrained set should have been much worse than that with the training pairs. But performance was accurate on this test. The monkeys' $87 \%$ untrained-set test performance was not significantly different from their $86 \%$ baseline performance. Also, the monkeys' test performance did not change materially over the 20 test sessions. These results were confirmed by a two-way repeated measures ANOVA of trial type (baseline, transfer) $\times$ session (1-20), which yielded no main effects of session $[F(19,57)<1]$ or trial type $[F(1,3)<1]$ and no interaction $[F(19,57)=1.4, p>.32]$.

The pigeons' $81 \%$ untrained-set test performance was accurate, but it was $10 \%$ lower than their $91 \%$ baseline performance $\left[F(1,3)=129, p<.002, \eta^{2}=.98\right]$. The pigeons' overall performance improved somewhat over the 20 test sessions $\left[F(19,57)=1.9, p<.04, \eta^{2}=.39\right]$, but there was no session $\times$ trial type interaction $[F(19,57)=$ $1.47, p>.13$ ], showing that the pigeons improved overall in the task rather than learned the testing pairs as a result of reinforcement. First-trial performance with pairs was $78 \%$ and $94 \%$ for pigeons and monkeys, respectively (all $t \mathrm{~s}>14$, all $p \mathrm{~s}<.002$, as compared with a $50 \%$ chance level), showing that accurate performance occurred before any learning by reinforcement with these pairs.

Accurate performance with the test pairs is best evaluated along with results from the novel-stimulus transfer test in the next section, because novel-stimulus transfer is the accepted measure of abstract-concept learning and is based on relational learning. Our previous studies using all possible training pairs did not show novel-stimulus transfer; therefore, none was expected here. Another purpose was to evaluate whether subjects in the present experiment would respond "different" when they were presented with any novel stimulus pair, which would thereby explain the untrained-set test results as a default or bias to respond "different."

\section{Novel-Stimulus Transfer}

Following the untrained-set test, pigeons and monkeys were tested with novel stimuli, and the results are shown in Figure 2. Neither monkeys (49.6\%) nor pigeons $(57.9 \%)$ showed any novel transfer that was significantly different from chance performance $[t(3)<1.9, p>.15]$. Differences between transfer and baseline were highly significant $\left[F(1,3)>58, p<.005, \eta^{2}>.95\right]$, as confirmed by a three-way repeated measures ANOVA of trial type (baseline, transfer) $\times \mathrm{S} / \mathrm{D}$ (same, different $) \times$ session (1-6), for each species. There were no trends in transfer performance across six test sessions for either species $[F(5,15)<1]$.
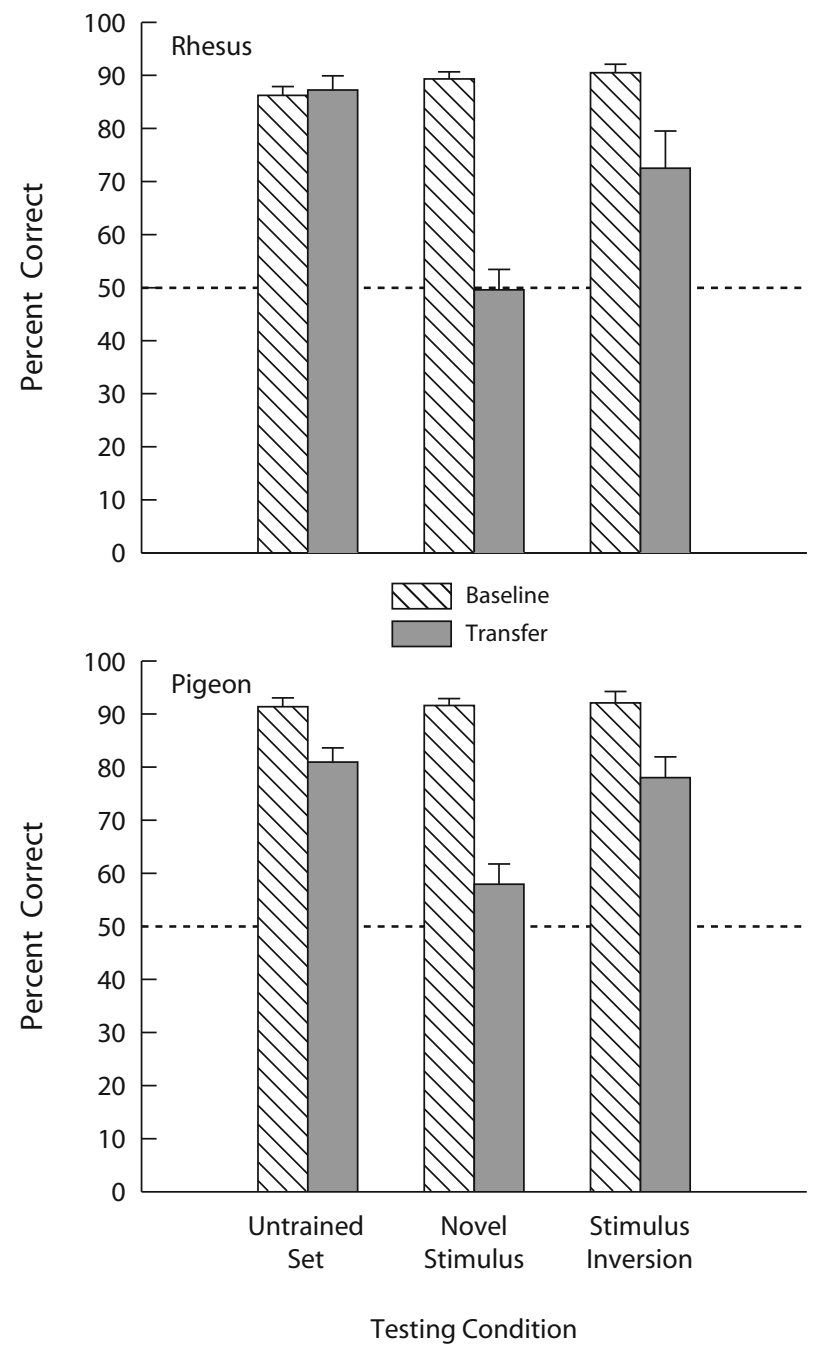

Figure 2. Baseline and transfer test results for rhesus monkeys and pigeons. The untrained-set test tested transfer to the stimulus pairs that are shown in the bottom of Figure 1. The novelstimulus test tested transfer to 60 novel-stimulus trials composed of 90 novel pictures that were selected to be different from the 8 training pictures. The stimulus-inversion test tested transfer to inversions of the pictures from the training stimulus pairs that are shown in the top of Figure 1. Error bars are standard errors of the means. Dotted lines are chance performance.

The absence of any statistical differences between same (pigeon, 46.7\%; rhesus, 66.7\%) and different performance (pigeon, $69.2 \%$; rhesus, $31.7 \%$ ) for either species on the novel-stimulus tests $[F(1,3)<1]$ shows that these subjects did not default to "different" when presented with unfamiliar stimuli or pairings, suggesting that these subjects compared the two stimuli during the untrained-set test and responded "different" on the basis of this comparison.

\section{Stimulus-Inversion Test}

The lack of novel-stimulus transfer shows that performance was in some way tied to the training stimuli, but accurate test performance with the untrained pairs shows that this S/D performance was not tied to the training 
stimuli through item-specific learning. Before venturing a conclusion about how these subjects were performing the S/D task, we conducted another test of item-specific learning in order to provide an independent converging measure or triangulation on this conclusion. For this test, we inverted the individual pictures of the training pairs that are shown in Figure 1 and tested these transformed training pairs in the manner of the previous tests.

Inversion of the individual stimuli of the training pairs should disrupt item-specific learning much more than does mirror-image rotation, because features at the bottom of pictures move to the top and vice versa. Subjects that are presented with an inverted sample stimulus would likely have their if-then chain of associative responses disrupted. Similarly, inverting the stimuli should discombobulate the whole appearance of a configural pattern that is produced by the training stimulus pair. Consequently, if-then chaining or gestalt configural pattern learningthe types of item-specific learning that are considered in this article - would likely be disrupted by inverting the stimuli. The basis of relational processing - judgments of whether the bottom picture is an identical match to the top picture - would remain intact, however. This was the logic and rationale of the stimulus-inversion test.

Results from the stimulus-inversion test are shown on the right in Figure 2. Inverting the stimuli had a somewhat detrimental effect on the transfer performance of both species, but not of the magnitude that would be expected if these species were learning the task item-specifically. The monkeys' $73 \%$ inversion-test performance, although $18 \%$ lower than their $91 \%$ baseline performance, was not significantly different from baseline performance $[F(1,3)=5.9, p>.09]$. In addition, the monkeys showed no effects across sessions or differences in same (74\%) versus different (71\%) performance on the test trials (all $F$ s $<1$ ). The pigeons' $78 \%$ inversion-test performance was significantly lower than their $92 \%$ baseline performance $\left[F(1,3)=21, p<.03, \eta^{2}=.88\right]$. Nevertheless, the pigeons' $78 \%$ stimulus-inversion transfer was significantly above chance performance $[t(3)=7.2, p<.01]$. The pigeons showed no effects across sessions or differences in same $(83 \%)$ versus different $(73 \%)$ performance on test trials (all $F \mathrm{~s}<1$ ). Once again, the lack of any tendency to respond "different" on these test trials supports the previously reached conclusion that performance on the untrained-set test was not the result of a bias to respond "different."

The pigeons' and monkeys' first-trial performance with the stimulus-inversion stimuli were $75 \%$ and $67 \%$, respectively $[t(3)>404, p<.0001$, as compared with $50 \%$ chance level]. As with the untrained-set test, these first-trial performances show accurate performance before any learning due to reinforcement to these pairs could have occurred.

\section{Implications for Item-Specific Learning}

The tests of the present study are among the first to test what animals learn when concept learning fails. The monkeys and pigeons performed accurately with pairs from the untrained-set test, including the first presentations of these untrained pairs. These results, coupled with the lack of a bias to respond "different" on the novel-stimulus and inverted-stimulus tests, suggest that accurate untrainedset performance was based on a comparison of the two pictures even though these pairs had not been seen before. If the subjects had learned if-then associative rules or the configural patterns for the training pairs, then they should not have performed well with the untrained-set pairs.

The validity of the tests and conclusions that are made here are supported by results from other experiments showing that pigeons do learn tasks item-specifically under other conditions. In one experiment, pigeons that were trained to either not peck the sample or peck it only once failed transfer on the untrained-set test in a matchingto-sample experiment and showed item-specific learning (gestalt-configural learning) of the training displays (Wright, 1997). In another experiment, pigeons that were trained with only three of the eight stimuli that were used in the present experiment learned the $\mathrm{S} / \mathrm{D}$ task in different ways. The subject of greatest interest to the present discussion failed both the untrained-set test and the stimulusinversion test, thereby showing that results from both of these tests converge on item-specific learning (Elmore, Wright, Rivera, \& Katz, 2009). Item-specific learning in the Elmore et al. experiment made sense, because that experiment had only 6 training displays to be learned, as opposed to the 40 displays in the present experiment. Itemspecific learning is more efficient the fewer the number of displays to be learned.

\section{A Case for Restricted-Domain Relational Learning}

That the subjects of the present experiment performed so well with inverted stimuli but failed with novel stimuli raises the issue of domain boundaries for relational learning. The inverted stimuli preserved the S/D relationship but distorted the stimuli. In the Elmore et al. (2009) study, two other pigeons tolerated their stimuli being swirled into something like a caramel concoction without too much disruption of performance. Apparently, distorting the stimuli is very different from substituting completely novel stimuli. Distorting the stimuli seems to preserve certain critical aspects of the visual domain (e.g., proportion of colors) while obliterating others (e.g., forms).

We conclude that these findings, taken together, make a case for restricted-domain relational learning - that is, being able to perform a task relationally within some limits that are circumscribed by the training stimuli. We should point out that restricted-domain relational learning is a novel proposal and is contrary to most theoretical treatments of abstract-concept learning. Item-specific learning has been the accepted explanation for what is learned when concept learning fails-but with no direct evidence to support it, except the failure of concept learning itself. Apparently, no one has seriously considered the possibility that relational learning can operate within a restricted portion of the stimulus domain that is defined by the characteristics of the training stimuli.

We hasten to add, however, that these conclusions regarding restricted-domain relational learning may not apply to 
other research showing failures of abstract-concept learning. The failure of concept learning in a fair proportion of other learning research may very well be based on itemspecific learning, such as in the experiments by Elmore et al. (2009) and Wright (1997). But one cannot know whether restricted-domain relational learning has occurred unless one specifically tests for item-specific learning.

The possibility of restricted-domain relational learning can be seen in human learning research. Even adult humans seem to suffer from similar restrictions in the domain within which they can properly apply relational learning (Chen \& Mo, 2004). Participants learning a relationship in one domain (e.g., three different-sized cups that were used to add or subtract water to obtain a target volume) had trouble seeing how a similar relationship would apply in another domain (e.g., three scale weights that were used to add or subtract coal). Comparison groups with the same amount of training but with training spread across several domains (e.g., length, area, volume) transferred accurately to the scale-weight domain. Children's relational shift, which has been found to be domain specific but to become more abstract as they age and learn more relationships, may be related (Gentner, 1988, 2003; Gentner \& Rattermann, 1991; Rattermann \& Gentner, 1998). This domain-specific relational shift seems to share aspects with the restricted-domain relational learning for animals that has been described in this article. The thought has occurred to us that some cases in which initial learning by children was concluded to be tied to specific features of the training stimuli may turn out to have been instances of restricted-domain relational learning instead of itemspecific learning. As we have seen from the results of the present study, restricted-domain relational learning can look a lot like item-specific learning unless specific tests are conducted to disrupt any item-specific learning.

\section{Conclusions}

The ways in which the domain might be restricted with regard to relational learning are not known and are likely to vary across individuals, as has been shown with pigeons in Elmore et al. (2009), but a lack of such specification is not unique to animal cognition experiments. In the human literature, for example, there is considerable agreement that "the relational shift reflects a qualitative change in children's mental representations ... can affect his or her reasoning ... [but] is nonetheless incomplete in that it does not provide an account of how this change takes place" (Doumas et al., 2008, p. 21). In any case, it is clear that with the monkeys and pigeons in our $\mathrm{S} / \mathrm{D}$ task, the domain expanded as the training set increased (i.e., novel-stimulus transfer increased). The domain is unlikely to expand in small steps along stimulus dimensions in the manner of stimulus generalization, however. Novel-stimulus transfer and the restricted-domain relational learning on which it is based appear to expand and encompass more diverse stimuli than would be realizable by any simple generalization process (Wright \& Katz, 2007). Indeed, we believe that this is the defining characteristic that makes abstractconcept learning or rule learning unique: The range of ac- curate application grows more rapidly than anything that might be expected from simple generalization. It now appears that expansion in the number of training items in some of our previous studies - in the process of producing more variations of the same/different rule and better transfer - had the effect of progressively expanding the stimulus domain within which the pigeons and monkeys could accurately apply the same/different relational rule.

\section{AUTHOR NOTE}

This research was supported by NIMH Grant MH-072616 and NSF Grant IBN-0316113. We thank Jacquelyne Rivera, Kent Bodily, Michelle Hernandez, Brad Sturz, and Kelly Schmidtke for their careful conducting of some of the tests. Correspondence concerning this article should be addressed to A. A. Wright, Department of Neurobiology and Anatomy, University of Texas Medical School at Houston, P.O. Box 20708, Houston, TX 77225 (e-mail: anthony.a.wright@uth.tmc.edu).

\section{REFERENCES}

CARTER, D. E., \& Werner, T. J. (1978). Complex learning and information processing by pigeons: A critical analysis. Journal of the Experimental Analysis of Behavior, 29, 565-601.

Chen, Z., \& Mo, L. (2004). Schema induction in problem solving: A multidimensional analysis. Journal of Experimental Psychology: Learning, Memory, \& Cognition, 30, 583-600.

Doumas, L. A. A., Hummel, J. E., \& Sandhofer, C. M. (2008). A theory of the discovery and predication of relational concepts. Psychological Review, 115, 1-43.

Elmore, L. C., Wright, A. A., Rivera, J. J., \& Katz, J. S. (2009). Individual differences: Either relational learning or item-specific learning in a same/different task. Learning \& Behavior, 37, 204-213. doi:10.3758/LB.37.2.204

GentNeR, D. (1988). Metaphor as structure mapping: The relational shift. Child Development, 59, 47-59.

Gentner, D. (2003). Why we're so smart. In D. Gentner \& S. GoldinMeadow (Eds.), Language in mind: Advances in the study of language and thought (pp. 195-235). Cambridge, MA: MIT Press.

Gentner, D., \& Rattermann, M. J. (1991). Language and the career of similarity. In S. A. Gelman \& J. P. Byrnes (Eds.), Perspectives on language and thought: Interrelations in development (pp. 225-277). New York: Cambridge University Press.

KatZ, J. S., \& WRight, A. A. (2006). Same/different abstract-concept learning by pigeons. Journal of Experimental Psychology: Animal Behavior Processes, 32, 80-86. doi:10.1037/0097-7403.32.1.80

Katz, J. S., Wright, A. A., \& Bachevalier, J. (2002). Mechanisms of same/different abstract-concept learning by rhesus monkeys (Macaca mulatta). Journal of Experimental Psychology: Animal Behavior Processes, 28, 358-368. doi:10.1037/0097-7403.28.4.358

LASHLEY, K. S. (1938). Conditional reactions in the rat. Journal of Psychology: Interdisciplinary \& Applied, 6, 311-324.

Premack, D. (1978). On the abstractness of human concepts: Why it would be difficult to talk to a pigeon. In S. H. Hulse, H. Fowler, \& W. K. Honig (Eds.), Cognitive processes in animal behavior (pp. 423451). Hillsdale, NJ: Erlbaum.

Premack, D. (1983a). Animal cognition. Annual Review of Psychology, 34, 351-362.

Premack, D. (1983b). The codes of man and beasts. Behavioral \& Brain Sciences, 6, 125-167.

Rattermann, M. J., \& Gentner, D. (1998). More evidence for a relational shift in the development of analogy: Children's performance on a causal-mapping task. Cognitive Development, 13, 453-478.

Siegler, R. S., \& Alibali, M. W. (2004). Children's thinking. Upper Saddle River, NJ: Prentice Hall.

Skinner, B. F. (1950). Are theories of learning necessary? Psychological Review, 57, 193-216.

SMITH, L. B. (1984). Young children's understanding of attributes and dimensions: A comparison of conceptual and linguistic measures. Child Development, 55, 363-380.

Sмiтн, L. B. (1989). From global similarities to kinds of similarities: 
The construction of dimensions in development. In S. Vosniadou \& A. Ortony (Eds.), Similarity and analogical reasoning (pp. 146-178). New York: Cambridge University Press.

Thомаs, R. K. (1980). Evolution of intelligence: An approach to its assessment. Brain, Behavior \& Evolution, 17, 454-472.

Wright, A. A. (1997). Concept learning and learning strategies. Psychological Science, 8, 119-123. doi:10.1111/j.1467-9280.1997 .tb00693.x

Wright, A. A., \& KatZ, J. S. (2006). Mechanisms of same/different concept learning in primates and avians. Behavioural Processes, $\mathbf{7 2}$, 234-254. doi:10.1016/j.beproc.2006.03.009
Wright, A. A., \& KatZ, J. S. (2007). Generalization hypothesis of abstract-concept learning: Learning strategies and related issues in Macaca mulatta, Cebus apella, and Columba livia. Journal of Comparative Psychology, 121, 387-397. doi:10.1037/0735-7036.121.4.387

Wright, A. A., Rivera, J. J., Katz, J. S., \& Bachevalier, J. (2003). Abstract-concept learning and list-memory processing by capuchin and rhesus monkeys. Journal of Experimental Psychology: Animal Behavior Processes, 29, 184-198. doi:10.1037/0097-7403.29.3.184

(Manuscript received January 27, 2009; revision accepted for publication May 18, 2009.) 